\title{
Acknowledgment to Referees for Dermatology 1996
}

The editor extends his gratitude and appreciation to the following reviewers whose comments and criticisms ensure the quality of the papers published in this journal.

Aliaga, A., Valencia, Spain

Aloi, F., Turin, Italy

Applegate, L.A., Lausanne, Switzerland

Auckenthaler, R., Geneve, Switzerland

Audetat, F.. Geneve, Switzerland

Baehni, P., Geneve, Switzerland Bagot, M., Créteil, France Baran, R., Cannes, France Baretton, G., München, Germany Barnhill, R.L., Boston, Mass., USA Baudraz-Rosselet, Fl., Lausanne, Switzerland Beani, J.C., Grenoble, France Benson, P.M., Washington, D.C., USA Beris, Ph., Geneve, Switzerland Bernard, Ph., Limoges, France Boehncke, W.H., Ulm, Germany Bongard, O., Geneve, Switzerland Bonifazi, E., Bari, Italy Bonnetblanc, J.M., Limoges, France Borisch, B., Geneve, Switzerland Borradori, L., Amsterdam, The Netherlands Böni, R., Zurich, Switzerland Bruckner-Tuderman, L., Münster, Germany Buezo, G.F., Madrid, Spain Burg, G., Zurich, Switzerland Büchner, S.A., Basel, Switzerland

Callen, J.P, Louisville, Ky., USA Camarasa, J.G., Barcelona, Spain Caputo, R., Milano, Italy Cesarini, J.P, Paris, France Chang, J.C., Carlsbad, Calif., USA Chizzolini, C, Geneve,

Switzerland Chosidow, O., Paris, France Claudy, A., Lyon, France Combemale. P., Lyon, France Cribier, B., Strasbourg, France Crovato, F, Chiavari, Italy Cunliffe, W.J., Leeds, United Kingdom Curtin-Cundy, D., Geneve, Switzerland

Eady, R.A.J., London, United Kingdom Elias, P.M., San Francisco, Calif., USA Eisner, P., Zurich, Switzerland Enk, A., Mainz, Germany

Farge, Dom., Paris, France Feldmann, R., Vienna, Austria Frances, C, Paris, France Frenk, E., Lausanne, Switzerland Furue, M., Tokyo, Japan

Garcia, J., Geneve, Switzerland

Geiger, J.-M., Lingolsheim, France

Gelmetti, C, Milano, Italy

Ghanem, G., Brussels, Belgium

Gorin, I., Paris, France

Griffiths, W.A.D., London, United Kingdom

Grimalt, R., Barcelona, Spain

Grob, J.J., Marseille, France

Grollier, J.F, Clichy, France

Gross, M., Lausanne, Switzerland

Grosshans, E., Strasbourg, France

Gschnait, F, Vienna, Austria

Guerne, PA., Geneve, Switzerland

Guillet, G., Brest, France

Gupta, M.A., London, Ont., Canada 
Hadengue, A., Geneve, Switzerland Hafner, J., Zurich, Switzerland Happle, R., Marburg, Germany Harms, M., Geneve, Switzerland Hauser, C, Geneve, Switzerland Heenen, M., Bruxelles, Belgium Henz-Czarnetzki, B.M., Berlin, Germany Hirschel, B., Geneve, Switzerland Hohl, D., Lausanne, Switzerland Hölzle, E.. Düsseldorf, Germany Hönigsmann, H., Vienna, Austria Humbert, Ph., Besançon, France

Ikeda, I., Fukuoka, Japan Itin, PH., Basel, Switzerland

Lachapelle, J.M., Brussels, Belgium

Lacour, J.-P, Nice, France

Lacour, M., London, United Kingdom

Landthaler, M., Regensburg, Germany

Lebbé, C, Paris, France

Leboit, P.E., San Francisco, Calif., USA

Le Poitevin, J.-P, Strasbourg, France

Limat, A., Bern, Switzerland

Mackie, R., Glasgow, United Kingdom Magistris, M.R., Geneve, Switzerland Mascaro, J.M., Barcelona, Spain Masgrau-Peya, E., Geneve, Switzerland Masouyé, I., Geneve, Switzerland McGovern, T.W., Aurora, Colo., USA Menter, A., Dallas, Tex., USA Mevorah, B., Tel-Aviv, Israel Miralbell, M., Geneve, Switzerland Mirimanoff, Geneve, Switzerland Morel, P., Paris, France Morlière, P., Créteil, France

Nishikawa, T, Tokyo, Japan Nordmann, Y., Colombes, France

Ortonne, J.P, Nice, France

Paller, A.S., Chicago, III., USA Panizzon, R.G., Lausanne, Switzerland Pasche, F, Geneve, Switzerland Pasini, W., Geneve, Switzerland Pechère, M., Geneve, Switzerland Péclard, N., Carouge, Switzerland Pepper, M., Geneve, Switzerland Perrenoud, D., Lausanne, Switzerland Piérard, G.E., Liège, Belgium Piletta, PA., Geneve, Switzerland Pinnell, S.R., Durham. N.C.. USA Pippione, M., Torino, Italy Plantin, P., Quimper, France Plewig, G., München, Germany Pollack, S.V.. Toronto, Ont., Canada

Dayer, J.M., Geneve, Switzerland Delescluse, J.P, Brussels, Belgium de Merloose, P., Geneva, Switzerland Dietrich, P.Y., Geneve, Switzerland Douglas-Jones, A.G., Cardiff,

United Kingdom Dubertret, L., Paris, France Dummer, R., Zurich, Switzerland Duvic, M., Houston, Tex., USA

James, R.W., Geneve, Switzerland Johnson, B.L., Portsmouth, Va., USA

Kanitakis, J., Lyon, France Kerl, H.,Graz. Austria King, L.E., Nashville, Tenn., USA Knobler, R., Vienna, Austria Krebs, A., Bern, Switzerland

Queille-Roussel, C, Nice, France

Ramelet, A.A., Lausanne, Switzerland Rebora, A., Genova, Italy Reichert, U,, Sophia Antipolis, France Reitamo, S., Helsinki, Finland Revuz, J., Créteil, France Rongioletti, F, Genova, Italy Rook, A.H., Philadelphia, Pa., USA

372

Roten, S., Geneve, Switzerland Roujeau, J.-C, Créteil, France Ruffieux, Ph., Geneve, Switzerland Rufli, Th., Basel, Switzerland Ruzicka, T., Düsseldorf, Germany

Saiag, Ph., Boulogne, France Salmon, I., Brussels, Belgium Samson, J., Geneve, Switzerland Sanchez Yus, E., Madrid, Spain Sato, K., Iowa City, Iowa, USA Schinzel, A., Zurich, Switzerland Schmied, E., La Chaux-de-Fonds, 
Switzerland Schmitt, D., Lyon, France Schurer, N., Düsseldorf, Germany Schwartz, R.A., Newark, N.J., USA Sehgal, V.N., Delhi, India

Shelley, W.B., Toledo, Ohio, USA Shimizu, S., Tokyo, Japan Shiohara, T, Tokyo, Japan Skaria, A., Geneve, Switzerland Skov, L., Hellerup, Denmark Sontheimer, R.D, Dallas, Tex., USA Souteyrand, P., Clermont-Ferrand, France Staughton, R.C.D, London, United Kingdom Surber, Ch., Basel, Switzerland

Tagami, H., Sendai, Japan Taïeb, A., Bordeaux, France Thestrup-Pedersen, K., Aarhus, Denmark Thiboutot, D, Pennsylvania, Pa., USA Tosti, A., Bologna, Italy Traupe, H., Münster, Germany Vahlquist, A., Linköping, Sweden Vahlquist, C, Linköping, Sweden Vaillant, L., Tours, France van de Kerkhof, P.C.M., Nijmegen,

The Netherlands Van Neste, D, Tournai, Belgium

Wallach, D, Paris, France Watanabe, T, Tokyo. Japan Westerhof, W., Amsterdam, The Netherlands Winkelmann, R.K., Scottsdale, Ariz., USA Wolf, R., Tel-Aviv, Israel Wolff, K., Vienna, Austria Woodley, D.T., Chicago, Ill., USA 